\title{
Relaciones entre fortalezas del carácter y percepción de apoyo social
}

\section{Relationships between character strengths and perception of social support}

\section{Relações entre forças de caráter e percepção do apoio social}

\author{
Ana Paula Porto Noronha ${ }^{1}$ \\ Elaine Nogueira Silva ${ }^{2}$ \\ Fabián J. M. Rueda ${ }^{3}$ \\ ${ }^{123}$ Programa de Pós-graduação Stricto Sensu em Psicologia, Universidade São Francisco. Brasil
}

\begin{abstract}
Resumen: El presente estudio tuvo como objetivo estudiar las relaciones entre fortalezas del carácter y la percepción del apoyo social a través de la Escala de Fortalezas del Carácter - EFC y de la Escala de Apoyo Social - EPSUS-IJ (Versión Infanto-Juvenil). Participaron de la investigación 293 adolescentes, con edades entre 14 y 17 años $(M=14.95, D T=3.038)$, de ambos sexos, siendo $67.8 \%$ del sexo femenino. Como resultado fue posible identificar que el factor 1 (Enfrentamiento de Problemas) contribuyó significativamente en 21 fortalezas. A su vez, en los factores Interacción Social y Afectividad presentaron coeficientes significativos en 14 y 12 fortalezas respectivamente. Los hallazgos se discuten con base en la literatura.
\end{abstract}

Palabras clave: psicología positiva, evaluación psicológica, apoyo social, fortalezas, adolescencia

\begin{abstract}
This study aimed to study the relations between character and social support, through the Characters Strengths Scale (EFC) and Social Support Scale - EPSUS-IJ (Youth version). 293 adolescents, 14 to 17 years $(M=14.95, S D=3.038)$ participated, $67.8 \%$ female. Results showed that the factor 1 (Contributing to Problems) EPSUS-IJ contributed significantly to explain 21 characters strengths. The second factor (Social Interaction) presented significant coefficients in 14 strengths, and factor 3 (Affectivity) in 12 strengths. The findings are discussed based on literature.
\end{abstract}

Keywords: positive psychology, psychological assessment, social support, strengths, adolescence

Resumo: O presente estudo teve por objetivo estudar as relações entre forças de caráter e percepção de suporte social, por meio da Escala de Forças de Caráter - EFC e da Escala de Suporte Social EPSUS-IJ (Versão Infanto-Juvenil). Participaram da pesquisa 293 adolescentes, com idades entre 14 e 17 anos $(M=14,95, D P=3,038)$, ambos os sexos, sendo $67.8 \%$ do sexo feminino. Como resultado foi possível identificar que o fator 1 (Enfrentamento de Problemas) contribuiu significativamente em 21 forças. Por sua vez, os fatores Interação Social y Afetividade apresentaram coeficientes significativos em 14 e 12 forças respetivamente. Os achados são discutidos à luz da literatura.

Palavras-chave: psicología positiva, avaliação psicológica, suporte social, forças, adolescência

Cómo citar este artículo:

Porto Noronha, A.P., Nogueira Silva, E., \& Rueda, F.J.M. (2018). Relaciones entre fortalezas del carácter y percepción de apoyo social. Ciencias Psicológicas, 12(2), 187-193. doi: https://doi.org/10.22235/cp.v12i2.1681.

Correspondencia: Ana Paula Porto Noronha. Rua Waldemar Cesar da Silveira, 105, Jardim Cura D'ars. Campinas - SP - Brasil. E-mails: ana.noronha8@gmail.com; elainenogueira.psi@gmail.com; fabian.rueda@usf.edu.br 


\section{Introducción}

Fortalezas del carácter son características relativamente estables que se pueden manifestar a través de comportamientos, pensamientos o sentimientos. Ellas deben ser auténticas para el individuo y permitir un funcionamiento próximo a su ideal (Peterson \& Seligman, 2004). Los autores elaboraron una clasificación con base en los resultados de una amplia investigación de la literatura, titulado Values in Action (VIA), que reúne 24 fortalezas distribuidas en seis virtudes, con el objetivo de describir claramente las potencialidades humanas.

Basados en el VIA fueron elaborados dos instrumentos, el Values in Action Inventory of Strengths (VIA-IS) y el Values in Action Inventory of Strengths for Youth (VIA-Youth), siendo que los dos tienen como objetivo evaluar las diferencias individuales en relación a las cualidades de las personas (Snyder \& Lopez, 2009). Los instrumentos fueron traducidos para varios idiomas, lo que originó muchas investigaciones, algunas de ellas con publicaciones más recientes (McGrath, 2015a; McGrath, 2015b; Proyer, Gander, Wellenzohn, \& Ruch, 2015; Ruch, Martínez-Martí, Proyer, \& Harzen, 2014; Ruch, Weber, Park, \& Peterson, 2014; Shoshani \& Slone, 2013; entre otros). En Brasil fueron traducidos al portugués y un estudio con una muestra brasileña fue publicado con el objetivo de realizar la adaptación transcultural, investigar la estructura interna y obtener datos demográficos (Seibel, DeSouza, \& Koller, 2015).

También en Brasil, con base en el modelo VIA Noronha y Barbosa (2016) construyeron la Escala de Fortalezas del Carácter. A partir de esa escala, esta investigación se propone verificar correlaciones entre los resultados del test y otras variables que miden el mismo constructo o constructos relacionados, así como con variables que miden constructos diferentes (American Educational Research Association, American Psychology Association, \& National Council on Measurement in Education - AERA, APA, \& NCME, 2014; Urbina, 2007). Tal evidencia será buscada correlacionando los constructos fortalezas de carácter y apoyo social, y la explicación teórica se sustenta en la comprensión de que el ambiente ejerce influencia en el fortalecimiento de las emociones y de las fortalezas del carácter, una vez que en un ambiente social promisorio y seguro, en el cual se estimulan las potencialidades, es prob- able que los individuos presenten más recursos para mejorar sus habilidades (Snyder \& Lopez, 2009). Para esos autores, niños y adolescentes que conviven en contextos sociales donde los aspectos negativos son evidentes, no desarrollan recursos de enfrentamiento y búsqueda de soluciones para sus problemas, y encaran la vida con poco entusiasmo. Campos (2004) realiza consideraciones semejantes, destacando que a medida que el individuo percibe ese apoyo, encuentra fortalezas para enfrentar situaciones adversas, lo que resulta en consecuencias positivas para su bienestar, como reducción del estrés, aumento de la autoestima y del bienestar psicológico.

Según Cobb (1976), el apoyo social se refiere a la ayuda recibida por los miembros del medio social del individuo, o sea, amigos, compañeros de escuela, trabajo y comunidad, pero también puede ser ofrecido por la familia. Las relaciones de apoyo pueden ser consideradas un factor positivo en el desarrollo de los individuos, teniendo en cuenta la necesidad de integración y satisfacción que el proceso de dar y recibir ayuda ocasiona, además del impacto en la autoestima (De Jong Gierveld \& Dykstra, 2008).

Incluso, con la intención de definir el constructo Cobb (1976) afirmó que para los individuos el apoyo social se relaciona a la creencia de ser amados, sentirse apreciados por su valor, y también la percepción de que las personas se preocupan con ellos. A su vez, Thois (1982) destacó que además de la dimensión afectiva mencionada anteriormente, el constructo 'apoyo social' presenta una dimensión llamada instrumental, en la cual el individuo percibe que las personas pueden ayudarlo por medio de recursos financieros y prácticos, como por ejemplo, préstamo de dinero, cuidar de un hijo por algunas horas, o cualquier otro tipo de ayuda. Rodriguez y Cohen (1998) afirmaron que una dimensión más debería formar parte del apoyo social, y sería el apoyo informativo, que se refiere a la posibilidad de recibir informaciones de otras personas que auxilien al individuo en el proceso de solución de sus problemas y de decisiones.

Una psicología con intervenciones positivas hacia la juventud es muy necesaria para el buen desarrollo de los jóvenes. Existen muchas evidencias de su eficacia en el aumento del bienestar, en la mejoría de la depresión (Proyer et al., 2015), y en el aumento del desempeño académico (Weber \& Rusch, 2012). No obstante, los autores se están dedicando preferencialmente a la determinación 
de las principales fortalezas de los individuos (Proyer et al., 2015), y no a la relación con otros constructos, lo que confirma la importancia de este estudio. Bastianello y Hutz (2016) también afirman que las investigaciones sobre asuntos relacionados a fortalezas del carácter, emociones y vínculos positivos, como optimismo y apoyo social, parecen ser poco abordados. Entre los temas investigados, se encuentran aquellos que se destinan a la construcción y validación de instrumentos, así como los que se destinan a la intervención (Renshaw \& Steeves, 2016).

A pesar de haber varios estudios con los instrumentos VIA (Toner, Haslam, Robinson, \& Williams, 2012), la relación con otros constructos todavía es incipiente, y se puede destacar la investigación de Shoshani y Slone (2013). En un estudio longitudinal, el objetivo de los autores fue examinar las asociaciones entre fortalezas del carácter, bienestar subjetivo, desempeño escolar de adolescentes y comportamiento social durante la transición para la Enseñanza Secundaria de escuelas públicas. Los resultados identificaron, especialmente en relación al comportamiento social, que las fortalezas bondad, amor y gratitud predicen ajuste social y pueden mejorar la capacidad de los adolescentes de mantener y establecer relaciones interpersonales y desarrollar identidades sociales.

Sobre la relación entre bienestar subjetivo, gratitud, esperanza/optimismo, amor y vitalidad, hubo correlaciones consideradas fuertes con satisfacción con la vida (Ruch, Martínez-Martí et al., 2014). Igualmente, al realizar una investigación con el objetivo de adaptar el VIA-Youth para la población de Alemania, Ruch, Weber et al. (2014) encontraron esas mismas fortalezas como siendo las más relacionadas a satisfacción con la vida.

En Brasil, utilizando la Escala de Fortalezas del Carácter (Noronha \& Martins, 2016), encontraron correlación moderada entre vitalidad, gratitud, esperanza/optimismo, perseverancia y amor con satisfacción con la vida. En estudio semejante, Oliveira, Nunes, Legal y Noronha (2016) encontraron relaciones entre bienestar subjetivo y las mismas fortalezas anteriores, además de humor y amor por el aprendizaje.

Con base en lo expuesto, y considerando la asociación entre las fortalezas del carácter con diferentes constructos, esta investigación tuvo la intención de buscar evidencias de validez para la Escala de Fortalezas del Carácter - EFC (Noronha $\&$ Barbosa, 2016) basadas en la relación con otras variables. Para eso, se eligió la Escala de Apoyo Social - EPSUS-IJ (Baptista \& Cardoso, 2013) para la asociación con la EFC.

\section{Método}

\section{Participantes}

Participaron de la investigación 293 adolescentes con edades entre 14 y 17 años $(M=14.95$, $D T=3.038$ ), de ambos sexos, siendo $67.8 \%$ del sexo femenino. Los participantes eran estudiantes del $8^{\circ}$ grado de Enseñanza Primaria al $3^{\circ}$ año de Enseñanza Secundaria. La muestra fue proveniente de cinco escuelas de la red pública de Enseñanza del interior del Estado de San Pablo, Brasil.

\section{Instrumentos}

- Escala de Fortalezas del Carácter - EFC (Noronha \& Barbosa, 2016). Desarrollada con base en el modelo Values in Action, se destina a la evaluación de las 24 fortalezas de carácter. Inicialmente fueron construidos 72 ítems, sometidos posteriormente a análisis de jueces y a un estudio piloto. Un ítem fue suprimido, y para investigar la estructura del instrumento se realizó un análisis de segunda orden, con las 24 fortalezas de carácter. Para determinar el número de factores que deberían ser extraídos, Noronha, Dellazzana-Zanon y Zanon (2015) utilizaron diferentes métodos de análisis. El coeficiente alpha (0.93) indicó alta confiabilidad.

- Escala de Percepción de Apoyo Social (Versión Infanto-Juvenil) - EPSUS-IJ (Baptista \& Cardoso, 2013). A partir de la descripción de Rodriguez y Cohen (1998), el objetivo de la escala es evaluar la percepción de niños y adolescentes del apoyo recibido por el contexto social, o sea, evaluar en qué medida los niños y adolescentes perciben sus relaciones sociales en los aspectos afectivos, de interacción social y enfrentamiento de problemas. Se trata de una escala compuesta por 23 ítems distribuidos en tres factores, siendo ellos: Enfrentamiento de Problemas ( 11 ítems, $\alpha=0.91$ ), relacionado a la percepción del apoyo de los integrantes de su red social en momentos de toma de decisiones, Interacción Social ( 5 ítems, $\alpha=0.88$ ), que evalúa la calidad de los relacionamientos de los sujetos con los individuos; y Afectividad (7 ítems, $\alpha=0.91$ ), relacionada al apoyo de orden emocional. 


\section{Procedimientos y consideraciones éticas}

Inicialmente se presentó el proyecto al Comité de Ética en Investigación de la Universidad San Francisco, y después de aprobado (CAAE 36085814.6 .0000 .5514 ) fue realizado el contacto con la Secretaría de Enseñanza de una ciudad del interior de San Pablo, para finalmente contactar a las instituciones de Enseñanza para la autorización de la recolección de los datos. Después de eso fueron programadas las aplicaciones.

Por tratarse de una investigación con adolescentes menores de 18 años, fueron pasadas todas las informaciones, orientaciones y procedimientos de recolección, así como solicitada la firma del Formulario de Consentimiento (TCLE), para que los alumnos entregaran a sus respectivos responsables. Después de recogidos los TCLEs, los alumnos fueron encaminados para una sala donde los instrumentos fueron aplicados. Primero se aplicó la EFC y después la EPSUS-IJ. La aplicación fue de forma colectiva, con duración de aproximadamente 45 minutos.

\section{Análisis de datos}

Para el análisis de los datos se utilizó la regresión lineal, por medio del software SPSS versión 22.0. Para eso, el método elegido fue el ENTER, considerando como variable dependiente cada una de las 24 fortalezas del carácter y, como independientes, las três dimensiones de la EPSUSIJ. Tal análisis fue utilizado para atender el objetivo del estudio (Hair, Tatham, \& Black, 2005).

\section{Resultados}

Con el propósito de cumplir el objetivo del estudio fue realizada la regresión lineal, tal como se ha informado anteriormente. Para eso fueron realizados análisis para prever las fortalezas del carácter, una de cada vez, a partir de las dimensiones de la EPSUS-IJ. Para cada una de las 24 regresiones, el Enfrentamiento de problemas (factor 1), la Interacción social (factor 2) y la Afectividad (factor 3 ) fueron seleccionados como variables independientes. La tabla 1 presenta los coeficientes de regresión resumidos.

Tabla 1

Coeficientes de Regresión Lineal de las Fortalezas del Carácter

\begin{tabular}{lccccr}
\hline Fortalezas del Carácter & $R$ & $R^{2}$ & Beta & \multicolumn{1}{c}{$t$} & \multicolumn{1}{c}{$P$} \\
\hline Gratitud & 0.12 & 0.11 & 0.35 & 3.90 & $<0.001$ \\
Curiosidad & 0.05 & 0.04 & 0.12 & 1.64 & 0.005 \\
Esperanza/Optimismo & 0.13 & 0.08 & 0.31 & 6.26 & $<0.001$ \\
Bondad & 0.05 & 0.04 & 0.21 & 5.70 & 0.006 \\
Justicia & 0.05 & 0.04 & 0.19 & 5.26 & 0.004 \\
Apreciación belleza & 0.09 & 0.08 & 0.16 & 5.59 & $<0.001$ \\
Perseverancia & 0.07 & 0.06 & 0.20 & 5.63 & $<0.001$ \\
Pensamiento Crítico & 0.08 & 0.07 & 0.02 & 5.51 & $<0.001$ \\
Modestia & 0.04 & 0.03 & 0.23 & 6.84 & 0.014 \\
Amor por el Aprendizaje & 0.07 & 0.06 & 0.27 & 4.77 & $<0.001$ \\
Espiritualidad & 0.09 & 0.08 & 0.33 & 4.35 & $<0.001$ \\
Autenticidad & 0.08 & 0.07 & 0.27 & 7.97 & $<0.001$ \\
Prudencia & 0.13 & 0.12 & 0.36 & 4.55 & $<0.001$ \\
Amor & 0.16 & 0.15 & 0.38 & 3.38 & $<0.001$ \\
Humor & 0.12 & 0.11 & 0.14 & 1.72 & $<0.001$ \\
Vitalidad & 0.14 & 0.13 & 0.25 & 0.46 & $<0.001$ \\
Sentido colectivo & 0.11 & 0.10 & 0.22 & 5.79 & $<0.001$ \\
Inteligencia emocional & 0.03 & 0.02 & 0.18 & 5.08 & 0.040 \\
Valentía & 0.10 & 0.09 & 0.33 & 6.45 & $<0.001$ \\
Liderazgo & 0.10 & 0.09 & 0.27 & 2.96 & $<0.001$ \\
Sensatez & 0.06 & 0.04 & 0.06 & 5.50 & 0.002 \\
Creatividad & 0.20 & 0.19 & 0.30 & 4.58 & $<0.001$ \\
Autorregulación & 0.02 & 0.01 & 0.17 & 3.56 & 0.108 \\
Perdón & 0.04 & 0.03 & 0.18 & 2.84 & 0.011 \\
\hline
\end{tabular}


De las 24 regresiones apenas la fortaleza de Autorregulación no fue significativa. En las 23 fortalezas restantes los coeficientes de determinación $\left(R^{2}\right)$ ajustado variaron de 0,02 (Inteligencia emocional) a 0,19 (Creatividad). Se observa también que 7 fortalezas fueron más bien predichas, a saber, Gratitud, Prudencia, Amor, Humor, Vitalidad, Sentido Colectivo y Creatividad. Además, la percepción de apoyo social representó 19\% de la variancia explicada de la fortaleza Creatividad, $15 \%$ del Amor y 13\% de la fortaleza Vitalidad. Para comprender mejor la contribución de las dimensiones de la EPSUS-IJ para la explicación de las fortalezas de carácter, es presentada la tabla 2 .

Los datos permiten identificar que el factor 1 (Enfrentamiento de Problemas) contribuyó significativamente en 21 fortalezas. Interacción Social, el segundo factor, presentó coeficientes significativos en 14 fortalezas, y el factor 3 (Afectividad) en 12 fortalezas.

\section{Discusión}

De acuerdo con McGrath (2015a) estudios como la presente investigación son relevantes, una vez que están dirigidos a las fortalezas de carácter, siendo que éstas están sujetas a diferencias léxicas, así como al contexto cultural, aunque sean reconocidas como características humanas universales. Por lo tanto, es necesario estudiar el modelo con distintas muestras.

La percepción favorable de apoyo social desempeña un papel protector frente a las dificultades encontradas por adolescentes (Thompson, Mazza, Herting, Randell, \& Eggert, 2005), pudiendo reducir efectos negativos sobre la salud (Nurullah, 2012), y en razón de eso, fue elegido como constructo a ser relacionado con la Escala de Fortalezas del Carácter. Para Procidano y Hellen (1983) el estudio de la percepción de apoyo social en adolescentes puede revelar informaciones importantes, pues dependiendo de la naturaleza

Tabla 2

Niveles de significancia relativos a la contribución de cada factor de la EPSUS-IJ para la explicación de las fortalezas del carácter

\begin{tabular}{|c|c|c|c|c|}
\hline \multirow[t]{2}{*}{ Factores de la EPSUS-IJ } & \multirow[t]{2}{*}{ Fortalezas del carácter } & \multicolumn{3}{|c|}{ Niveles de significancia } \\
\hline & & Factor 1 & Factor 2 & Factor 3 \\
\hline \multirow[t]{7}{*}{ Factor 1 - Factor 2 - Factor 3} & Prudencia & $p<0.001$ & $p=0.001$ & $p=0.006$ \\
\hline & Apreciación belleza & $p=0.022$ & $p<0.001$ & $p=0.006$ \\
\hline & Esperanza/Optimismo & $p<0.001$ & $p<0.001$ & $p<0.001$ \\
\hline & Creatividad & $p<0.001$ & $p<0.001$ & $p<0.001$ \\
\hline & Amor & $p<0.001$ & $p<0.001$ & $p=0.001$ \\
\hline & Liderazgo & $p<0.001$ & $p=0.002$ & $p=0.002$ \\
\hline & Sentido Colectivo & $p<0.001$ & $p=0.008$ & $p<0.001$ \\
\hline \multirow[t]{3}{*}{ Factor 1 - Factor 2} & Autenticidad & $p<0.001$ & $p<0.001$ & \\
\hline & Valentía & $p<0.001$ & $p<0.001$ & \\
\hline & Religiosidad & $p<0.001$ & $p=0.015$ & \\
\hline \multirow[t]{3}{*}{ Factor 1 - Factor 3} & Perseverancia & $p=0.006$ & & $p=0.001$ \\
\hline & Vitalidad & $p<0.001$ & $p=0.003$ & \\
\hline & Humor & $p=0.046$ & & $p<0.001$ \\
\hline \multirow[t]{8}{*}{ Factor 1} & Amor al aprendizaje & $p<0.001$ & & \\
\hline & Justicia & $p=0.008$ & & \\
\hline & Bondad & $p=0.001$ & & \\
\hline & Inteligencia emocional & $p=0.014$ & & \\
\hline & Modestia & $p=0.001$ & & \\
\hline & Autorregulación & $p=0.017$ & & \\
\hline & Gratitud & $p<0.001$ & & \\
\hline & Perdón & $p=0.014$ & & \\
\hline \multirow[t]{3}{*}{ Factor 2 - Factor 3} & Curiosidad & & $p=0.011$ & $p=0.007$ \\
\hline & Pensamiento crítico & & $p=0.050$ & $p<0.001$ \\
\hline & Sensatez & & $p=0.001$ & $p=0.010$ \\
\hline
\end{tabular}


de las relaciones establecidas con los pares, son observados cambios a lo largo de su desarrollo, lo que impacta negativa o positivamente en la percepción de apoyo social.

En relación a cuánto los participantes de este estudio perciben que sus relaciones sociales poseen aspectos afectivos y auxilian en el enfrentamiento de problemas, eso previó como mayor destaque siete fortalezas. Amor (valoriza relaciones con los otros, en las cuales hay intercambio y cariños mutuos, y prioriza estar cerca de las personas), Sentido de colectividad (trabaja bien en grupo, colabora y es leal) y Humor (hace reír a las personas sistemáticamente, valoriza los aspectos positivos de las situaciones) están bastante volcados para el contacto con el otro, lo que justifica los resultados. Unido a eso, Gratitud (estar consciente que cosas buenas suceden) también favorece la relación con los acontecimientos de la vida y con las personas.

En otra medida, las fortalezas Creatividad (intención de hacer las cosas de una manera diferente), Vitalidad (vivir con entusiasmo y energía) y Prudencia (tener cautela en las decisiones, para no correr riesgos indebidos) parecen ser necesarias para establecer relaciones favorables. Tal como destacado por Snyder y Lopez (2009), si el contexto social carece de modelos y afectos positivos, los jóvenes tendrán menos recursos para encarar la vida con entusiasmo y resolver sus problemas. En esa misma dirección, los autores endosan que el ambiente ejerce gran influencia en el fortalecimiento de las fortalezas de carácter, y desde esta perspectiva, un ambiente prometedor y seguro tiende a estimular el desarrollo de las potencialidades, de modo que el individuo tiende a presentar más recursos para perfeccionar sus habilidades.

Ruch, Martínez-Martí et al. 2014 desarrollaron una investigación con el objetivo de adaptar el VIA-Youth para la población de Alemania. Sus resultados corroboran parcialmente los del presente estudio, pues las fortalezas de carácter esperanza, gratitud, amor y vitalidad se correlacionaron positivamente con la satisfacción con la vida. Los resultados de Shoshani y Slone (2013) revelaron que, en relación al comportamiento social las fortalezas interpersonales (entre ellas bondad, amor y gratitud) son predictoras del ajuste social y, además de eso, pueden mejorar la capacidad de los adolescentes para mantener y establecer relaciones interpersonales y desarrollar identidades sociales y sentimiento de pertenencia, también reafirmando parte de los resultados aquí encontrados. Los autores endosan que el apoyo social y un sentimiento de pertenencia al grupo se asocian con bienestar psicológico.

En relación al mejor predictor, la dimensión 1 de la EPSUS-IJ, relacionada a la percepción sobre cuánto recibe apoyo de los integrantes de su red social en momentos de toma de decisiones, fue el más frecuente. Tal dato es pertinente, considerando las afirmaciones de Bastianello y Hutz (2016), al destacar que personas con alta percepción de apoyo social presentan mejor ajuste emocional. Por fin, las fortalezas de carácter pueden ser fortalecidas y programas que tienen por objeto ampliarlas han obtenido efectos satisfactorios (Proyer, et al., 2015; Wagner \& Ruch, 2015).

\section{Consideraciones finales}

Los resultados indicaron que la percepción de apoyo social recibido por los individuos en su red social en momentos de toma de decisiones está relacionado con las fortalezas de carácter. Así, las fortalezas de carácter son entendidas como factores de protección de los jóvenes, tal como lo señalado por Peterson y Seligman (2004). Esta investigación puede colaborar en la comprensión social de los fenómenos emocionales, familiares y sociales en los sujetos, a la vez que la investigación científica de las potencialidades humanas es relevante para que se pueda contribuir con el desarrollo del saber psicológico.

Una limitación del estudio se refiere a la muestra, prioritariamente representada por jóvenes de escuelas públicas de una ciudad del interior del estado de San Pablo. De esa forma, como agenda de investigación se ve la necesidad de ampliar las variables analizadas y contar con una muestra más representativa. Verificar la relación entre jóvenes y padres también puede sugerir otras reflexiones importantes.

\section{Referencias}

American Educational Research Association, American Psychological Association, National Council on Measurement in Education - AERA, APA, \& NCME (2014). Standards for Educational and Psychological Testing. New York: American Educational Research Association.

Baptista, M. N., \& Cardoso, H. F. (2013). Escala de Percepção do Suporte Social (Versão Infanto-Juvenil): relatório técnico não publicado. Universidade São Francisco: Itatiba.

Bastianello, M. R., \& Hutz, C. S. (2016). Otimismo e suporte social em mulheres com câncer de mama: uma revisão sistemática. Psicologia: teoria e prática, 18(2), 19-33. doi: 10.15348/1980-6906/PSICOLOGIA.V18N2P19-33. 
Campos, E. P. (2004). Suporte Social e Família. En: J. Mello Filho (Org.), Doença e família (pp. 141-161). São Paulo: Casa do Psicólogo.

Cobb, S. (1976). Social support as a moderator of life stress. Psychosomatic Medicine, 38(5), 300-314. doi: 10.1097/00006842-197609000-00003.

De Jong Gieverld, J., \& Dykstra, P. A. (2008). Virtue is its own reward? Support-giving in the family and loneliness in middle and old age. Ageing and Society, 28(2), 271-287. doi: 10.1017/S0144686X07006629.

Hair, J. F., Tatham, R. L., \& Black, C., (2005). Análise Multivariada de Dados. Porto Alegre: Bookman.

McGrath, R. E. (2015a). Measurement Invariance in Translations of the VIA Inventory of Strengths. European Journal of Psychological Assessment, 1, 1-8. doi: 10.1027/1015-5759/a000248.

McGrath, R. E. (2015b). Integrating psychological and cultural perspectives on virtue: The hierarchical structure of character strengths. The Journal of Positive Psychology, 10(5), 407-424. doi: 10.1080/17439760.2014.994222.

Noronha, A. P. P., \& Barbosa, A. J. G. (2016). Escala de Forças e Virtudes. En C. Hutz (Org.), Avaliação em Psicologia Positiva (pp. 42-53). São Paulo: Hogrefe.

Noronha, A. P. P., Dellazzana-Zanon, L. L., \& Zanon C. (2015). Internal Structure of the Strengths and Virtues Scale in Brazil. Psico-USF, 20(2), 229-235. doi: 10.1590/141382712015200204.

Noronha, A. P. P., \& Martins, D. F. (2016). Associações entre forças de caráter e satisfação com a vida: estudo com universitários. Acta Colombiana de Psicologia, 19(2), 83-89.

Nurullah, A. S. (2012). Received and provided social support: a review of current evidence and future directions. American Journal of Health Studies, 27(3), 173-188.

Oliveira, C., Nunes, M. F. O., Legal., E., \& Noronha, A. P. P. (2016). Subjective Well-Being: Linear Relationships to Character Strengths. Avaliação Psicológica, 15(3), 177-185.

Peterson, C., \& Seligman, M. E.P. (2004). Character Strengths and Virtues: A Handbook and Classification. Washington, DC: American Psychological Association.

Procidano, M. E., \& Heller, K. (1983). Measures of perceived social support from friends and from family: Three validation studies. American Journal of Community Psychology, 11(1), 1-24. doi: 10.1007/BF00898416.

Proyer, R. T., Gander, F., Wellenzohn, S., \& Ruch, W. (2015). Strengths-based positive psychology interventions: A randomized placebo-controlled online trial on long-term effects for a signature strengths- vs. a lesser strengthsintervention. Frontiers in Psychology, 9(456), 1-14. doi: 10.3389/fpsyg.2015.00456.

Renshaw, T., \& Steeves, R. M. O. (2016). What good is gratitude in youth and schools? A systematic review and meta-analysis of correlates and intervention outcomes. Psychology in the Schools, 53(3), 286-305. doi: 10.1002/ pits.21903.
Rodriguez, M., \& Cohen, S. (1998). Social support. En: H. Friedman (Org.), Encyclopedia of Mental Health (pp. 535-544). New York: Academic Press.

Ruch, W., Martínez-Martí, M. L., Proyer, R. T., \& Harzen, C. (2014). The Characters Strengths Rating Form (CSRF): Development and initial assessment of a 24-item rating scale to assess character strengths. Personality and Individual Differences, 68, 53-58.

Ruch, W., Weber, M., Park, N., \& Peterson, C. (2014). Character strengths in children and adolescents: Reliability and initial validity of the German Values in Action Inventory of Strengths for Youth (German VIA-Youth). European Journal of Psychological Assessment, 30(1), 57-64. doi: 10.1027/1015-5759/a000169.

Seibel, B. L., DeSouza, D., \& Koller, S. H. (2015). Adaptação Brasileira e Estrutura Fatorial da Escala 240-item VIA Inventory of Strengths. Psico-USF, 20(3), 371-383. doi: 10.1590/1413-82712015200301.

Shoshani, A., \& Slone, M. (2013). Middle School Transition from the Strengths Perspective: Young adolescents' Character Strengths, Subjective Well-Being and School Adjustment. Journal of Happiness Studies, 14(4), 11631181. doi: 10.1007/s10902-012-9374-y.

Snyder, S. J., \& Lopez, C. R. (2009). Psicologia Positiva: uma abordagem cientifica e prática das qualidades humanas. Porto Alegre: Artmed.

Thoits, P. A. (1982). Conceptual, methodological and theoretical problems in studying social support as a buffer against life stress. Journal of Health and Social Behavior, 23(2), 145-159.

Thompson, E. A., Mazza, J. J., Herting, J. R., Randell, P. B., \& Eggert, L. L. (2005). The mediating roles of anxiety, depression, and hopelessness on adolescent suicidal behaviors. Suicide Life Threat Behavior, 35(1), 14-34. doi: 10.1521/suli.35.1.14.59266.

Toner, E., Haslam, N., Robinson, J., \& Williams, P. (2012). Character strengths and wellbeing in adolescence: Structure and correlates of the Values in Action Inventory of Strengths for Children. Personality and Individual Differences, 52, 637-642. doi: 10.1016/j.paid.2011.12.014.

Urbina, S. (2007). Fundamentos da testagem psicológica. Porto Alegre: Artmed.

Wagner, L., \& Ruch, W. (2015). Good character at school: Positive classroom behavior mediates the link between character strengths and school achievement. Frontiers in Psychology, 6(610), 1-13. doi: 10.3389/ fpsyg.2015.00610.

Weber, W., \& Ruch, W. (2012). The role of a good character in 12-year-old school children: Do character strengths matter in the classroom? Child Indicators Research, 5, 317-334. doi: 10.1007/s12187-011-9128-0. 